This is the peer reviewed version of the following article:

López-Muñoz G.A., Estévez M.-C., Vázquez-García M., Berenguel-Alonso M., Alonso-Chamarro J., Homs-Corbera A., Lechuga L.M.. Gold/silver/gold trilayer films on nanostructured polycarbonate substrates for direct and label-free nanoplasmonic biosensing. Journal of Biophotonics, (2018). 11. e201800043: - . 10.1002/jbio.201800043,

which has been published in final form at https://dx.doi.org/10.1002/jbio.201800043. This article may be used for non-commercial purposes in accordance with Wiley Terms and Conditions for Use of Self-Archived Versions. 


\title{
Gold/Silver/Gold Trilayer Films On Nanostructured Polycarbonate Substrates For Direct And Label-Free Nanoplasmonic Biosensing
}

\author{
Gerardo A. López-Muñoz,, ${ }^{a, b}$ M.-Carmen Estévez,, ${ }^{\text {ba }}$ Marc Vázquez-García, ${ }^{a}$ Miguel \\ Berenguel-Alonso, ${ }^{\mathrm{c}}$ Julián Alonso-Chamarro, ${ }^{\mathrm{c}}$ Antoni Homs-Corbera, ${ }^{\mathrm{a}}$ and Laura M. \\ Lechuga $^{a, b}$
}

\begin{abstract}
${ }^{a}$ Nanobiosensors and Bioanalytical Applications Group (NanoB2A), Catalan Institute of Nanoscience and Nanotechnology (ICN2), CSIC and BIST, Campus UAB, Bellaterra, 08193 Barcelona, Spain

${ }^{\mathrm{b}}$ CIBER-BBN Networking Center on Bioengineering, Biomaterials and Nanomedicine, Spain

'Sensors \& Biosensors Group, Department of Chemistry, Autonomous University of Barcelona, Edifici Cn, 08193 Bellaterra, Catalonia, Spain
\end{abstract}

\begin{abstract}
Ultrasmooth gold/silver/gold trilayer nanostructured plasmonic sensors were obtained using commercial Blu-ray optical discs as nanoslits-based flexible polymer substrates. A thin gold film was used as an adhesion and nucleation layer to improve the chemical stability and reduce the surface roughness of the overlying silver film, without increasing ohmic plasmon losses. The structures were physically and optically characterized and compared with nanostructures of single gold layer. Ultrasmooth and chemically stable trilayer nanostructures with a surface roughness $<0.5 \mathrm{~nm}$ were obtained following a simple and reproducible fabrication process. They showed a Figure of Merit (FOM) value up to 69.2 $\mathrm{RIU}^{-1}$ which is significantly higher (more than 95\%) than the gold monolayer counterpart. Their potential for biosensing was demonstrated by employing the trilayer sensor for the direct and label-free detection of CRP protein biomarker in undiluted urine achieving a LOD in the pM order.
\end{abstract}

Keywords: Blu-ray disc; nanoplasmonic devices; silver nanoslits; nucleation layer; flexible substrate. 
Surface Plasmon Resonance (SPR) phenomena in metallic nanostructures have undergone extensive development due to their ability to confine light at the nanoscale and the possibility of coupling and tuning different photonic effects (e.g. Fabry-Perot cavity, Wood's anomalies or Fano Resonances). These characteristics confer on SPR the potential for widespread use in applications related to photon management [1], microscopy [2], surface-enhanced spectroscopy [3] or sensing [4].

In recent years, the development of nanoplasmonics has been carried out using mainly gold nanostructures due to their high plasmonic performance and excellent chemical stability [5]. However, due to its dielectric properties, silver has the highest plasmonic activity within all noble metals when used to build nanostructures and is also more economically attractive [6]. The main obstacles faced by silver-based nanostructures when dealing with different plasmonic applications are related to the silver's low chemical stability and poor wettability (i.e. the deposited atoms have greater tendency to bind to each other rather than to the substrate atoms, favoring three dimensional islands growth and as consequence increasing rough surface) on dielectric substrates which tend to diminish the substrates performance and limit their applications [6-8]. In order to overcome these challenges two main approaches have been explored to generate chemically stable and flat silver plasmonic films on dielectric substrates: 1) the addition of a protective surface layer (i.e. self-assembled monolayers (SAM) [9], oxides and other metals [10]); and 2) the use of a nucleation layer (i.e. germanium [11], chromium + gold [10] and polymers [12]). The addition of a protective thin gold film (Ag/Au bilayer system) can increase the chemical stability. However, under certain oxidative environments such as saline solutions, the chemical stability and reproducibility for long term use can be also affected. This can be partly related to the low wettability (which implies high roughness) of silver on dielectric substrates and the mismatch adhesion of silver with different materials $[8,10]$. To overcome these difficulties, the use of nucleation layers has been also proposed, but most of the materials employed (i.e. germanium, titanium, chromium) increase ohmic plasmon losses, thereby diminishing the 
final performance $[13,14]$. Recently, another approach using a thin gold nucleation layer between a chromium adhesion layer and the upper silver layer has been demonstrated to successfully generate flat silver plasmonic films with improved chemical stability on glass substrates creating a four layer system $(\mathrm{Cr} / \mathrm{Au} / \mathrm{Ag} / \mathrm{Au})[9,10]$.

The use of polymer-based substrates can increase the potential applications of plasmonic nanostructures, as the flexibility these materials can confer expand their use, for example, enabling the direct integration of sensors in the human body, or for solar energy harvesting, among others [15,16]. As recently reported [17], the fabrication of plasmonic gold sensors using commercial Blu-ray discs as a flexible nanostructured polycarbonate polymer substrate (substrate with concentric periodic nanoslits of $320 \mathrm{~nm}$ period, $160 \mathrm{~nm}$ width and $\approx$ $20 \mathrm{~nm}$ depth) is a simple and highly reproducible process. Efficient deposition of thin gold films can be accomplished without the need of an adhesion layer (i.e. germanium, chromium, titanium) that can negatively affect the plasmonic performance in different ways $[13,14]$

Based on these considerations, we have fabricated plasmonic sensor devices that combine trilayer $(\mathrm{Au} / \mathrm{Ag} / \mathrm{Au})$ films and the precise nanostructured arrays of the commercial polycarbonate Blu-ray discs (see Supporting Information, SI, for details). The nanostructures were characterized and assessed from a biosensing perspective. A total metallic film thickness of $100 \mathrm{~nm}$ was selected for the fabrication of the devices as previous studies demonstrated that optimal plasmonic performance occurred at thicknesses between 50 and $120 \mathrm{~nm}$. At metallic layers below $50 \mathrm{~nm}$, the plasmonic reflectance spectra lose sharpness due to the strong interaction of plasmonic waves with the underlying substrate, whereas for metallic layers above $100 \mathrm{~nm}$, no significant change in the plasmonic reflectance spectra is observed [17,18]. For the adhesion/nucleation layer, different Au layer thicknesses were evaluated (from $0 \mathrm{~nm}$ to $5 \mathrm{~nm}$ ), which seems to be sufficient to improve the adhesion of the silver layer while minimizing alterations to the plasmonic propagation due to a strong optical absorption of the adhesion/nucleation layer [19]. On the other hand, different thicknesses of 
top Au layer (5 $\mathrm{nm}$ to $25 \mathrm{~nm}$ ) were evaluated to improve the chemical stability of the substrate under high oxidative media $\left(\mathrm{UV} / \mathrm{O}_{3}\right.$ oxidation and a high salt content media) commonly used in biosensing assays. For comparison we also fabricated Ag/Au bilayer systems without the Au nucleation layer.

The bare and the multilayered nanostructured surfaces were first characterized by Atomic Force Microscopy (AFM). Figure 1 shows the surface roughness and the profile. Surface roughness values obtained in root-mean-square (RMS) were $0.21,0.36,1.29$ and $0.65 \mathrm{~nm}$ for bare Blu-ray, $\mathrm{Au}, \mathrm{Ag} / \mathrm{Au}$ and $\mathrm{Au} / \mathrm{Ag} / \mathrm{Au}$ surfaces, respectively. For the $\mathrm{Au} / \mathrm{Ag} / \mathrm{Au}$ surface, a $2 \mathrm{~nm} \mathrm{Au}$ nucleation layer increases the adhesion of $\mathrm{Ag}$ to the substrate, reducing the stratification and therefore decreasing the surface roughness compared to the $\mathrm{Ag} / \mathrm{Au}$ surface (0.44 $\mathrm{nm}$ vs $1.08 \mathrm{~nm}$, by subtracting the surface roughness of the bare nanostructured substrate) [9].The roughness value for the $\mathrm{Au} / \mathrm{Ag} / \mathrm{Au}$ nanostructured polymeric substrate (0.44 nm) was lower than the one previously reported for $\mathrm{Cr} / \mathrm{Au} / \mathrm{Ag} / \mathrm{Au}$ [9] and $\mathrm{Ge} / \mathrm{Ag}$ [8] multilayer films, being all of them fabricated on flat glass substrates. These results emphasize that also for polymeric substrates, ultrasmooth plasmonic $\mathrm{Ag}$ based nanostructures can be fabricated without using adhesion/nucleation materials which significantly increase ohmic plasmon losses (i.e. germanium, chromium, titanium) [17].

The trilayered $\mathrm{Au} / \mathrm{Ag} / \mathrm{Au}$ nanostructured polymer substrate was also optically characterized with reflectance measurements, collecting spectra at different angles of incident light $\left(30^{\circ}\right.$ $\left.70^{\circ}\right)$ with a Transverse Mode (TM) polarized broadband light in air $(n=1.00)$ and water $(\eta=1.33)$ (see SI, Figure S1). The observed shift in the resonance peak position $\left(\lambda_{\mathrm{SPP}}\right)$ to higher wavelengths and the narrowing of resonant linewidths with high incident angles is correlated with the generation of Fano resonances [17]. By increasing the angle of the incident light, the optical energy scattered from one nanostructure can be collected by neighboring nanostructures decreasing radiative loss and increasing the plasmon lifetime, as previously reported [17] for Au nanoslits. The sensors integrated with a single channel flow cell (see SI, Figure S2 for the sensing set-up) was used to evaluate the optical behavior of 
the multilayer sensors. The optical effect of different adhesion/nucleation Au layer thicknesses $(0,2$ and $5 \mathrm{~nm})$ was evaluated by observing the shift in the plasmonic resonance peak position ( $\left.\lambda_{\mathrm{SPP}}\right)$ when injecting different refractive index (RI) solutions (i.e. glycerol solutions between $4.2 \mathrm{mM}$ and $68 \mathrm{mM}$ ). As shown in the SI, Figure S3, for the $\mathrm{Au} / \mathrm{Ag} / \mathrm{Au}$ system, an adhesion/nucleation $\mathrm{Au}$ thickness layer close to $2 \mathrm{~nm}$ seems not to affect the plasmonic propagation due to a strong optical absorption compared to a thicker $\mathrm{Au}$ layer (i.e. $5 \mathrm{~nm}$ ) and other adhesion/nucleation materials (i.e. Ge, $\mathrm{Cr}$ and Ti) $[8,9,20]$.

The optical characterization results were contrasted with those calculated from FDTD simulations (see SI for details). As can be observed in Figure 2a there is a good agreement between the calculated and the experimental reflectance spectra, with a narrower resonant linewidth for the $\mathrm{Au} / \mathrm{Ag} / \mathrm{Au}$ substrate compared to the Au substrate. As previously discussed, the dielectric properties of $\mathrm{Ag}$ provide nanostructures with higher plasmon field enhancements and narrower FWHM spectra compared of those with only a Au monolayer. To evaluate the theoretical plasmon field enhancement in $\mathrm{Au} / \mathrm{Ag} / \mathrm{Au}$ and $\mathrm{Au}$ substrates, we analyzed the electric field distribution calculated from FDTD simulations. Figures $2 \mathrm{c}$ and $2 \mathrm{~d}$ illustrate the plasmon enhancement in the electric field distributions of the $\mathrm{Au} / \mathrm{Ag} / \mathrm{Au}$ and the Au substrates, respectively. The higher plasmonic activity owing to the addition of a silver layer in $\mathrm{Au} / \mathrm{Ag} / \mathrm{Au}$ substrates is noticeable with an increase in the intensity of the optical fields compared to Au substrates [6,9].

The effect of the top Au layer thickness in the chemical stability of the trilayered nanostructured plasmonic sensor was evaluated under high oxidative conditions $\left(\mathrm{UV}-\mathrm{O}_{3}\right.$ oxidation and a high salt content media) commonly used in biosensing assays and it was compared with bilayer sensors as reference (no Au nucleation/adhesion layer). Multilayer sensors with three different top Au layer thicknesses (5, 15 and $25 \mathrm{~nm}$ ) were placed in a $\mathrm{UV} / \mathrm{O}_{3}$ generator for $10 \mathrm{~min}$. A top $\mathrm{Au}$ layer thickness $\geq 15 \mathrm{~nm}$ provides a significant protection against oxidation. Moreover, the lack of the $2 \mathrm{~nm}$ nucleation layer (i.e. bilayer sensors) results in a significant degradation even with a top layer of $15 \mathrm{~nm}$, which makes 
clear the necessity of including this layer in the design (see SI, Figure S4). The stability under aqueous oxidative media was evaluated by integrating the sensors with the flow cell and performing real-time tracking of the resonance peak position $\left(\lambda_{\mathrm{SPP}}\right)$ (see SI, Figure S5). A high salt content solution (PBS $100 \mathrm{mM}$ with $1.4 \mathrm{M} \mathrm{NaCl}$ ) was continuously flowed (see SI for details). A steady baseline ( $\left.\Delta \lambda_{\mathrm{SPP}}\right)$ is observed for the trilayer $\mathrm{Au} / \mathrm{Ag} / \mathrm{Au}$ system as in the case of single Au layer substrates, which confirms its stability over time. Under the same experimental conditions, the bilayer system $(\mathrm{Ag} / \mathrm{Au})$ suffers a pronounced change in the $\Delta \lambda_{\text {SPP. }}$. As expected the improvement in adhesion and as consequence, the decrease of roughness of silver using the thin Au adhesion/nucleation layer, is correlated with the improved chemical stability of the $\mathrm{Au} / \mathrm{Ag} / \mathrm{Au}$ substrate compared to $\mathrm{Ag} / \mathrm{Au}$ counterpart [9].

The performance of the trilayer plasmonic device for sensing applications was also evaluated with different glycerol solutions. Main parameters were extracted after injection of the different solutions: bulk sensitivity, FWHM and the resultant figure of merit (FOM) at different angles of incident light. The measurements were done also in real time, keeping as running buffer a constant flow of $\mathrm{H}_{2} \mathrm{O}(30 \mu \mathrm{L} / \mathrm{min})$. The results were compared to those previously obtained for the Au plasmonic sensor [17]. Figure $2 \mathrm{~b}$ shows a comparison of the sensing performance parameters for both plasmonic devices. The $\mathrm{Au} / \mathrm{Ag} / \mathrm{Au}$ device resulted in better overall performance compared to Au layered substrates at all the incident angle tested, being $70^{\circ}$ the best one as previously reported [17]. Although the enhancement in sensitivity was around $12 \%$ (bulk sensitivity of $476 \mathrm{~nm} \cdot \mathrm{RIU}^{-1}$ vs $425 \mathrm{~nm} \cdot \mathrm{RIU}^{-1}$ in $\mathrm{Au}$ ), the narrowing of the peak (with a FWHM reduced approximately 57\%, from $12 \mathrm{~nm}$ to $7 \mathrm{~nm}$ ) led to an enhancement of the FOM of a factor of two (from 34.9 to $69.2 \mathrm{RIU}^{-1}$ for the trilayered device).

This improvement was evaluated also by a biosensing assay on the trilayered $\mathrm{Au} / \mathrm{Ag} / \mathrm{Au}$ nanostructured sensor. A direct assay based on the attachment of specific antibodies and the detection of the corresponding target protein was considered (see Supporting Information for experimental details). The selected protein, C-reactive protein (CRP) is a 
well-known and valuable biomarker related with inflammation and infection processes. $\mathrm{Au} / \mathrm{Ag} / \mathrm{Au}$ and $\mathrm{Au}$ nanostructured surfaces were modified by forming a self-assembled monolayer (SAM) with carboxylic acid, which was activated and further reacted with the specific antibody for CRP. The immobilization of the antibody was monitored in real time, as can be seen in the SI, Figure S6. The detection of CRP at different concentrations (from 25 to $1000 \mathrm{ng} / \mathrm{mL}$ ) shows a good dose-response (see Figure 3a). We were able to achieve a LOD of $2.6 \mathrm{ng} / \mathrm{mL}(20.8 \mathrm{pM})$ and a LOQ of $9.1 \mathrm{ng} / \mathrm{mL}(72.9 \mathrm{pM})$, respectively (see the calibration curves in the SI, Figure S7) which represents an improvement in biosensing performance compared to the Au single layer sensor (LOD of $3.7 \mathrm{ng} / \mathrm{mL}$ and a LOQ of 12.9 $\mathrm{ng} / \mathrm{mL}$ ). The higher plasmonic activity using a silver layer improves the biosensing performance of the device $[9,10]$. The viability of measuring biological samples like undiluted urine was also assessed with these sensors. In this case, in order to minimize non-specific adsorptions from components present in the complex media, an additional blocking step was included in the biofuncionalization protocol. A solution of poly(L-lysine) poly(ethylene glycol) (PLL-PEG) $(0.5 \mathrm{mg} / \mathrm{mL})$ was added to the antibody-immobilized sensor, as this reagent has previously demonstrated its effectiveness as antifouling agent [22]. Similar shift was observed for the same concentration of CRP in buffer before and after blocking (see Figure 3b (black and red lines), which confirms that this step does not affect the ability of the antibody to bind its target. The injection of pure undiluted urine and undiluted urine including a non-specific protein (i.e. BSA) resulted in no background $\left(\Delta \lambda_{S P P}=0\right)$ (see Fig. $3 b$, green and magenta lines), which confirms the lack of nonspecific binding onto the biofunctionalized surface. Finally, the same CRP concentration in pure urine also resulted in the same signal obtained in buffer (same $\Delta \lambda_{\mathrm{SPP}}=0$ ) (blue line in Fig. 3b). All these measurements confirm the feasibility of directly measuring urine samples. Sensorgrams for different CRP concentrations summarized in the Figure $3 c$ and resulting calibration curve for CRP in urine showed comparable sensitivities to the ones obtained with standard buffer conditions demonstrating that urine components did not hinder the immunochemical reaction (see Figure 3d). While the LOD in PBS was $2.6 \mathrm{ng} / \mathrm{mL}$, a close value was reached with pure urine 
$(\mathrm{LOD}=3.5 \mathrm{ng} / \mathrm{mL}(27.9 \mathrm{pM})$, and $\mathrm{a} \mathrm{LOQ}=12.3 \mathrm{ng} / \mathrm{mL}(97.7 \mathrm{pM}))$. Overall these results reflect the promising performance of this kind of easy-to-fabricate flexible/polymer nanostructured $\mathrm{Au} / \mathrm{Ag} / \mathrm{Au}$ plasmonic substrates, with high potential to implement low cost competitive devices for biosensing applications.

In conclusion, we report the fabrication of a simple and ultrasmooth (surface roughness $<0.5$ nm) $\mathrm{Au} / \mathrm{Ag} / \mathrm{Au}$ nanostructured plasmonic sensor using commercial Blu-ray optical discs as nanoslits-based flexible polymer substrates. In contrast to other strategies, our approach takes advantage of the high adhesion of gold in polycarbonate to improve the wettability and reduce the surface roughness in the $\mathrm{Au} / \mathrm{Ag} / \mathrm{Au}$ nanostructured plasmonic sensors, without increasing ohmic plasmon losses and using only a thin gold film as adhesion/nucleation layer for the overlying silver film. This novel trilayer structure achieves better performance in terms of sensitivity and resolution over the purely Au counterpart. Furthermore, we can obtain Ag containing plasmonic devices which are chemically stable over time. The labelfree biosensing capability of the proposed $\mathrm{Au} / \mathrm{Ag} / \mathrm{Au}$ plasmonic structure was evaluated by performing real-time detection of the CRP protein biomarker in PBS buffer and in pure undiluted urine, obtaining sensitivities in both cases in the pM order. Biodetection results showed a significant improvement when compared to Au plasmonic surfaces, indicating the potential to obtain chemical stable and ultrasmooth silver/gold flexible polymer nanostructured sensors for direct and label-free biosensor applications.

\section{Supporting Information}

Additional supporting information may be found in the online version of this article at the publisher's website.

The materials and methods are detailed in the Supporting Information: the fabrication and integration of the nanostructured plasmonic device, the surface and optical characterization, FDTD simulations and the biosensing measurements (sensor surface biofunctionalization and CRP detection assays). Figures related are also included. 


\section{Acknowledgements}

GL acknowledges financial support from CONACYT 225362 scholarship. We thank Dr. Raul Pérez Rodríguez from ICN2 Nanofabrication Facility for their technical support in the AFM measurements. We acknowledge the financial support from PreDICT project (Programa estatal de investigación, desarrollo e innovación orientada a los Retos de la Sociedad, TEC2016-78515-R). The NanoB2A is a consolidated research group (Grup de Recerca) of the Generalitat de Catalunya and has support from the Departament d'Universitats, Recerca i Societat de la Informació de la Generalitat de Catalunya (2014 SGR 624). The ICN2 is funded by the CERCA programne / Generalitat de Catalunya. The ICN2 is supported by the Severo Ochoa programme of the Spanish Ministry of Economy, Industry and Competitiveness (MINECO, grant no. SEV-2013-0295) 


\section{References}

1. A. J. Smith, C. Wang, D. Guo, C. Sun, and J. Huang, Nat. Commun. 2014, 5, 5517.

2. B. Chen, A. Wood, A. Pathak, J. Mathai, S. Bok, H. Zheng, S. Hamm, S. Basuray, S. Grant, K. Gangopadhyay, P. V. Cornish, and S. Gangopadhyay, Nanoscale 2016, 8, 12189.

3. S. Basuray, A. Pathak, S. Bok, B. Chen, S. C. Hamm, C. J. Mathai, S. Guha, K. Gangopadhyay, and S. Gangopadhyay, Nanotechnology 2016, 28, 025302.

4. K. L. Lee, J. B. Huang, J. W. Chang, S. H. Wu, and P. K. Wei, . Sci. Rep. 2015, 5, 8547.

5. G. A. Lopez, M.-C Estevez,; M. Soler, and L. M. Lechuga, Nanophotonics 2017, 6, 123.

6. Zhu, X.; Zhuo, X.; Li, Q.; Yang, Z.; Wang, J. Gold Nanobipyramid-Supported Silver Nanostructures with Narrow Plasmon Linewidths and Improved Chemical Stability. Adv. Funct. Mater. 2016, 26, 341.

7. W. Chen, M. D. Thoreson, S. Ishii, A. V. Kildishev, and V. M. Shalaev, Opt. Express 2010,18, 5124.

8. V. J. Logeeswaran, N. P. Kobayashi, M. S. Islam, W. Wu, P. Chaturvedi, N. X. Fang, S.Y. Wang, and R. S. Williams, Nano Lett. 2009, 9, 178.

9. Z. Wang, Z. Cheng, V. Singh, Z. Zheng, Y. Wang, S. Li, L. Song, and J. Zhu, Anal. Chem.2014, 86, 1430.

10. Z. Cheng, Z. Wang, D. E. Gillespie, C. Lausted, Z. Zheng, M. Yang, and J. Zhu, Anal. Chem. 2015, 87, 1466.

11. W. Chen, M. D. Thoreson, S. Ishii, A. V. Kildishev, and V. M. Shalaev, Opt. Express. $2010,18,5124$.

12. L. Ke, S. C. Lai, H. Liu, C. K. N. Peh, B. Wang, and J. H. Teng, ACS Appl. Mater. Interf. 2012, 4, 1247.

13. P. Wróbel, T. Stefaniuk, M. Trzcinski, A. A. Wronkowska, A. Wronkowski, and T. Szoplik, ACS Appl. Mater. Interf. 2015, 7, 8999.

14. M. A. Otte, M.-C. Estévez, L. G. Carrascosa, A. B. González-Guerrero, L. M. Lechuga, and B. Sepúlveda, J. Phys. Chem. C. 2011, 115, 5344. 
15. D. Shir, Z. S. Ballard, A. Ozcan, IEEE J. Sel. Top. Quantum Electron. 2016, 22, 4600509.

16. J. G. Smith, J. A. Faucheaux, and P. K. Jain, Nano Today 2015, 10, 67.

17. G. A. Lopez-Muñoz, M.-C Estevez, E.C. Gutierrez Pelaez, A. Homs-Corbera, M.C. García-Hernández, J.I. Imbaud, and L. M. Lechuga, Biosens. Bioelectron. 2017, 96, 260.

18. H. Guner, E. Ozgur, G. Kokturk, M. Celik, E. Esen, A. E. Topal, S. Ayas, Y. Uludag, C. Elbuken,; A. Dana, Sens. Actuators B Chem. 2017,239, 571.

19. A. Kossoy, V. Merk, D. Simakov, K. Leosson, S. Kéna-Cohen, and S. A. Maier, Adv. Opt. Mater. 2015, 3, 71.

20. H. Liu, B. Wang, E. S. Leong, P. Yang, Y. Zong, G. Si, J. Teng, and S. A. Maier, ACS Nano 2010, 4, 3139.

21. T. Stefaniuk, P. Wróbel, P. Trautman, and T. Szoplik, App. Opt. 2014, 53, B237.

22. M. Soler, M.-C. Estevez, M. Álvarez, M. A. Otte, B. Sepulveda and L. M. Lechuga, Sensors 2014, 14, 2239. 


\section{Figure Legends}

Figure 1. AFM characterization: (a) AFM section analysis of four different layers of Blu-ray based nanostructured substrate: bare Blu-ray substrate, monolayered Au layered substrate, bilayered $\mathrm{Ag} / \mathrm{Au}$ substrate and trilayered Au/Ag/Au substrate. (b) AFM image of the bare Bluray based substrate. (c) AFM image of the $100 \mathrm{~nm}$ Au layer Blu-ray based substrate. (c) AFM image of the $85 \mathrm{~nm} \mathrm{Ag} / 15 \mathrm{~nm}$ Au layer Blu-ray based substrate. (d) AFM image of the $2 \mathrm{~nm} \mathrm{Au} \mathrm{/} 83 \mathrm{~nm} \mathrm{Ag} \mathrm{/15} \mathrm{nm} \mathrm{Au} \mathrm{layer} \mathrm{Blu-ray} \mathrm{based} \mathrm{substrate.}$

Figure 2. Optical characterization and FDTD Simulations of the $\mathrm{Au} / \mathrm{Ag} / \mathrm{Au}$ plasmonic structure. (a) Evaluated and simulated optical reflectance spectra under TM-polarized light for the fabricated $\mathrm{Au}$ and $\mathrm{Au} / \mathrm{Ag} / \mathrm{Au}$ nanostructured plasmonic structure. (b) Variation of the bulk sensitivity, FWHM and FOM respectively as function of the angle of the incidence light for the $\mathrm{Au}$ and $\mathrm{Au} / \mathrm{Ag} / \mathrm{Au}$ plasmonic devices; the left axis indicates the bulk sensitivity $\left(\mathrm{nm} \cdot \mathrm{RIU}^{-1}\right)$ and the right axis indicates the FWHM and FOM for the plasmonic devices; the error bar indicates the standard deviation of three measurements. Simulated electric field distribution for the Au substrate (c) and Au/Ag/Au substrate (d) under TM-polarized light.

Figure 3. Biosensing experiments for the $\mathrm{Au}$ and $\mathrm{Au} / \mathrm{Ag} / \mathrm{Au}$ sensors. (a) Sensorgrams showing the detection of the target CRP protein at different concentrations (from 25 to 1000 $\mathrm{ng} / \mathrm{mL}$ ) in PBS buffer; (b) Blocking step and urine effect. Sensorgrams showing the detection of CRP (200 ng/mL) in PBS without PLL-PEG blocking (black); the detection of CRP (200 $\mathrm{ng} / \mathrm{mL}$ ) in PBS after PLL-PEG blocking (red); nonspecific binding of undiluted urine (green); nonspecific binding of control protein (BSA, $500 \mu \mathrm{g} / \mathrm{mL}$ ) in urine (magenta);detection of CRP $(200 \mathrm{ng} / \mathrm{mL}$ ) in undiluted urine (blue); (c) Sensorgrams showing the detection of the target CRP protein at different concentrations (from 25 to $1000 \mathrm{ng} / \mathrm{mL}$ ) in undiluted urine. (d) CRP calibration curves in PBS and undiluted urine. The error bars reflect the standard deviation (SD) from two measurements. 


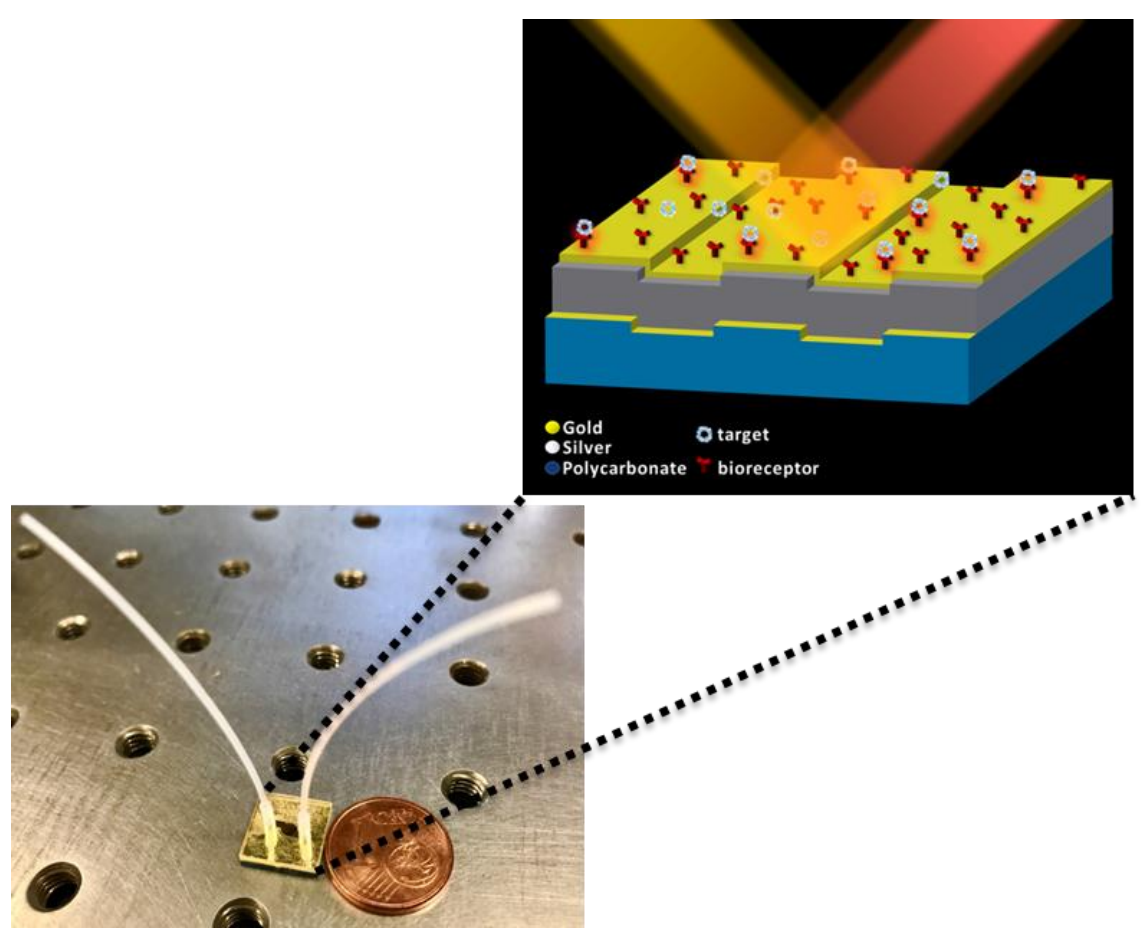

Gold/silver/gold nanostructured sensors based on commercial Blu-ray optical discs as substrates and with integrated microfluidics have been designed and optimized. Ultrasmooth and chemically stable trilayer nanoslit-based nanostructures were obtained following a simple and reproducible fabrication process. The direct and label-free detection of C-reactive protein (CRP) biomarker in undiluted urine was achieved with a LOD in the pM order. 


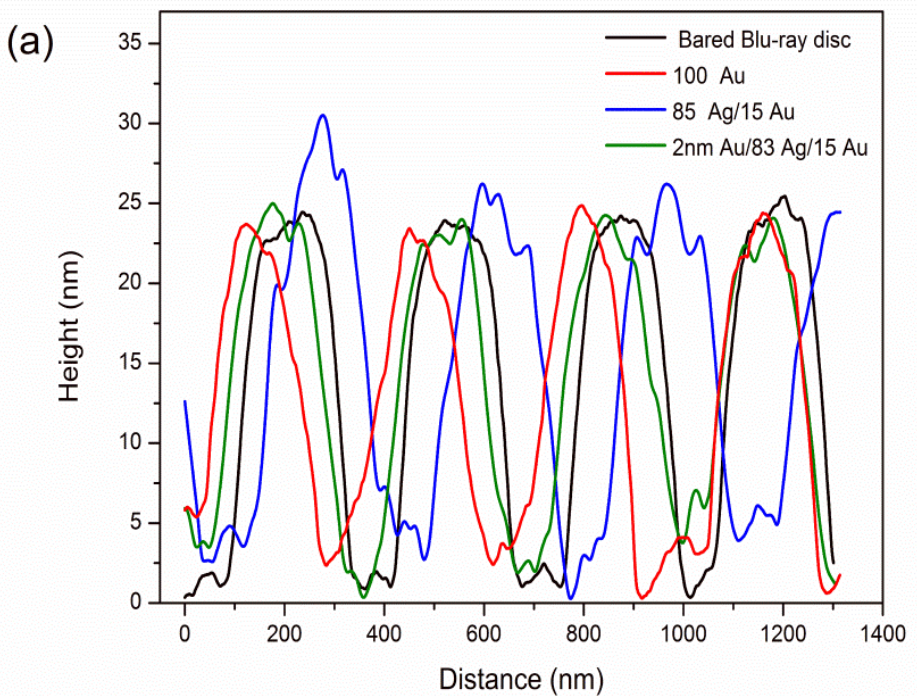
(b) $\sigma_{\text {RMS }}=0.21 \mathrm{~nm}$
(c) $\sigma_{\mathrm{RMS}}=0.36 \mathrm{~nm}$
(d) $\sigma_{\text {RMS }}=1.29 \mathrm{~nm}$
(e) $\sigma_{\mathrm{RMS}}=0.65 \mathrm{~nm}$
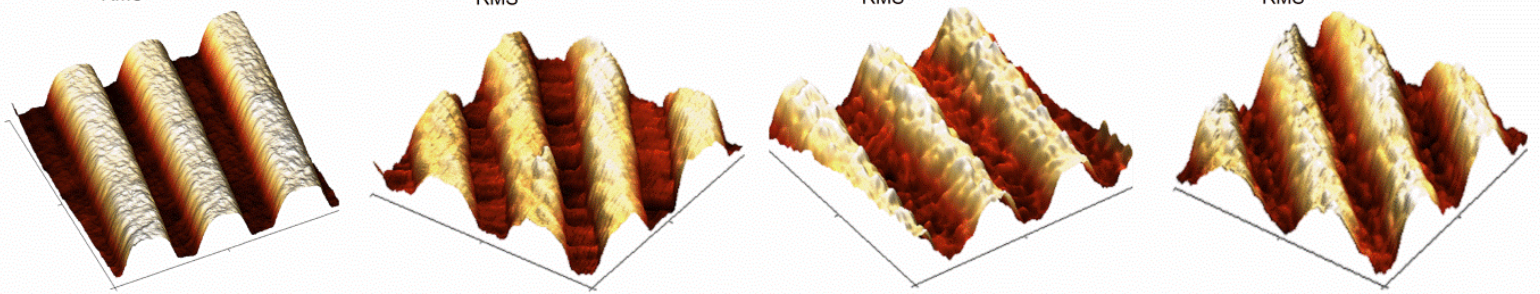

Figure 1 

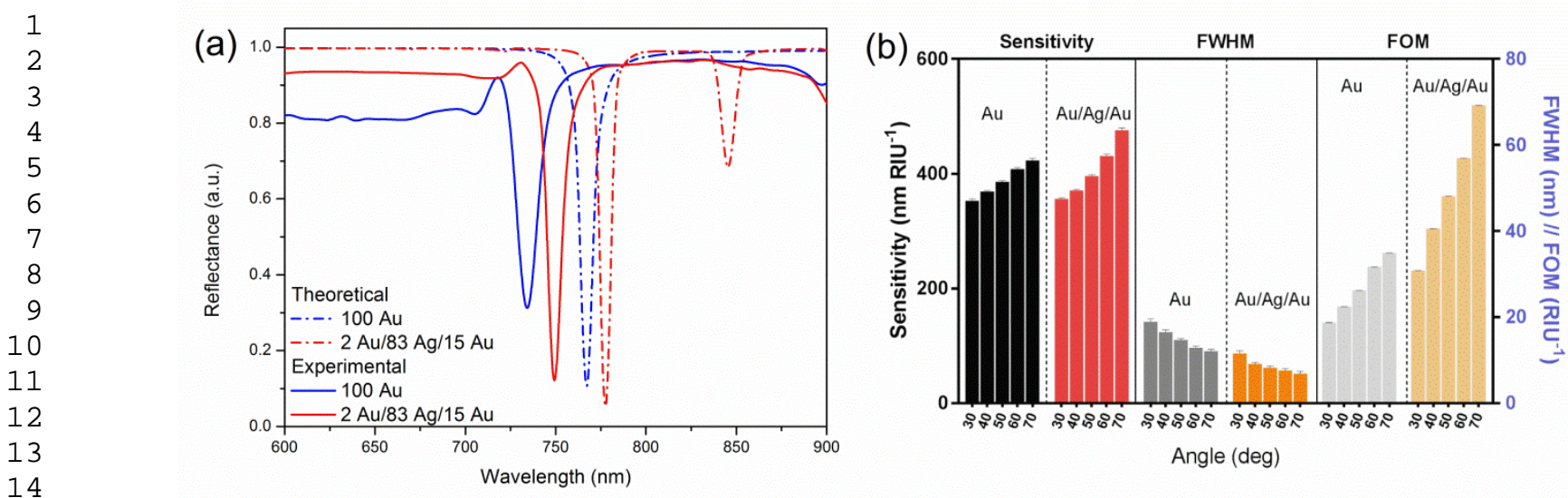

(c)

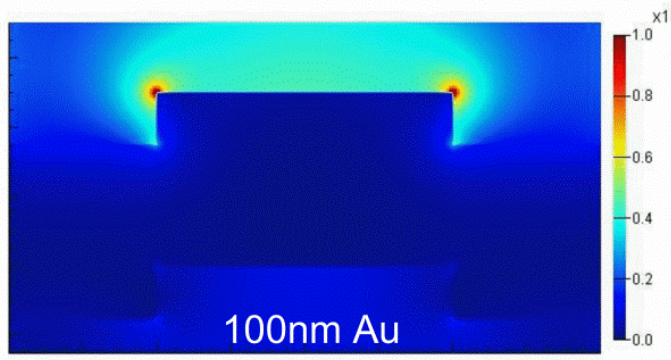

(d)

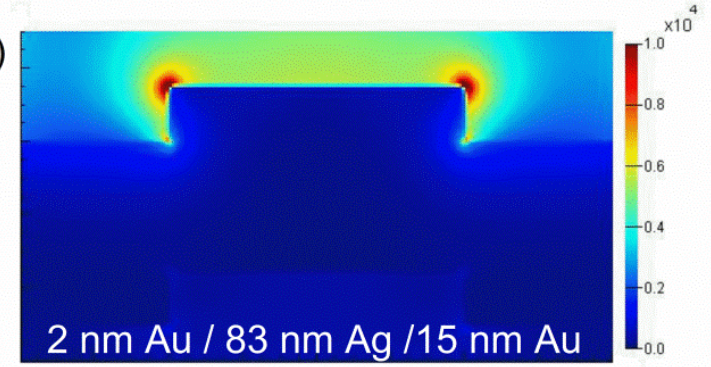

Figure 2 

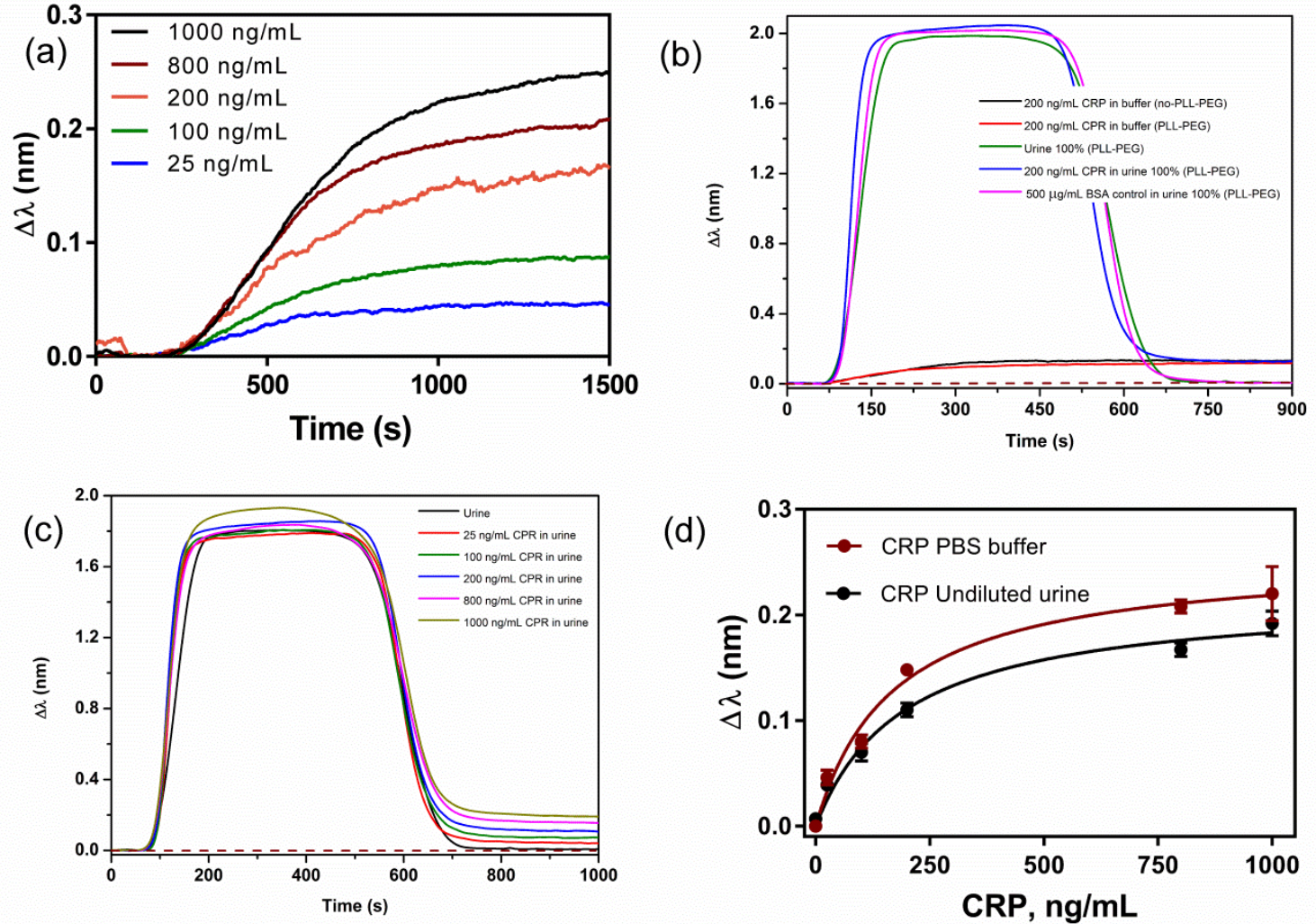

(d)

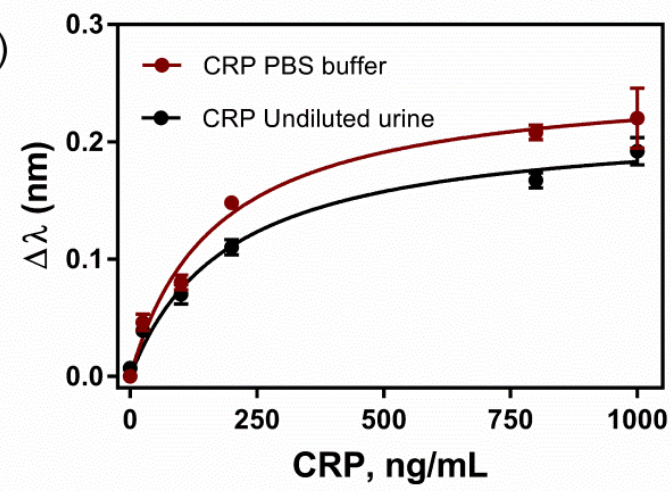

Figure 3 